\title{
Making it Work: Vocational Peer Mentors for Emerging Adults with Serious Mental Health Conditions
}

Tiansitions RTC

The Transitions RTC and Thresholds Young Adult Program (YAP) developed a supported employment/ education model based on the Individual Placement and Support ${ }^{1}$ model (IPS) and added a vocational peer mentor for emerging adults with serious mental health conditions (SMHC). This model is still being developed, but preliminary research has identified several guidelines that could be helpful for others thinking about implementing peer mentors into their vocational services for emerging adults with SMHC.

\section{Tips for Implementing Vocational Peer Mentors for Emerging Adults}

\section{Fully Integrate Peer Mentors into Service Team}

- Leadership should promote the value of peer mentors in order for the rest of the team to understand and meaningfully integrate the peer mentors into the delivery of vocational services.

- Clearly define the role of the peer mentors in relation to other members of the team.

- Set up mechanisms (e.g. group team meetings) to ensure that the peer mentor is working in collaboration with the team.

\section{Develop Peer Mentors}

- Adopt or develop training for peer mentors. We developed a 40-hour training on the IPS model, how the mentor can support vocational goals within IPS, group and individual engagement techniques, and how to tell one's story in an empowering way.

- Provide training and supervision on how to build and maintain strong relational boundaries with mentees, including how to maintain confidentiality.

- Define the role of the peer mentors in relation to the mentees. Partner with organizational leaders to develop clear peer mentor policies and practices.

\section{Support Mentee-Mentor Interactions}

- Support in-person community meetings (for example, supply gift cards to coffee shops).

- Provide peer mentors with cell-phone (or reimbursement) \& email account.

- Support peer mentor travel costs to meet mentees closer to their work, school, or home.

- Support "off-hours" connections between peer mentors \& mentees if necessary.

\section{Support Peer Mentors}

- Provide weekly group supervision meetings to manage relational boundaries.

- Help mentors identify when their own mental health issues arise and connect them with supports.

- Continue training based on arising topics (e.g., conflict resolution; self-care; mental health symptoms and treatment).

- Check-in with peer mentors about their own vocational development, struggles, and successes.

- Empower mentors to develop advocacy skills to be used in team meetings, both for themselves and for mentees. 


\section{The Model We Developed}

WHAT DID WE DO? The Transitions RTC and Thresholds Young Adult Program (YAP) collaborated to adapt the evidence-based Individual Placement and Support (IPS) ${ }^{1}$ model of supported employment for 16-21 year olds with serious mental health conditions. This adaptation included the addition of Supported Education services and the addition of near-age vocational peer mentors with similar life experiences (e.g., mental health diagnosis, treatment, and system involvement).

WHO WERE THE PEER MENTORS? Vocational peer mentors were ages 20 to 30 and were either employed, in an education program, or both. "Peer" was defined as having similar life experiences such as mental health diagnoses, treatment experience, and system involvement. The role of the vocational peer mentors was to engage the mentees in vocational services, provide encouragement in vocational pursuits, and coach and model professionalism.

HOW DID THEY “MENTOR?” After being matched with 4-6 mentees, peer mentors met individually with mentees in the community each week. They also participated in vocational groups, a vocational staff team meeting, and clinical supervision. Mentors provided emotional and informational support to the mentee as they pursued their vocational goals. They supported the exploration of jobs, careers, and schools, coached professionalism, and promoted engagement with the education and employment specialists.

WHAT WERE THE CHALLENGES TO ADDING PEER MENTORS TO IPS? Peer mentor turnover was high until older peer mentors (over age 25) were recruited who were further along in their own vocational attainment and who did not maintain the same peer groups as their mentees. Finding mutually available times for peer mentors and mentees to connect proved challenging due to the many school, work, and mental health treatment commitments that both peer mentors and mentees have. Finally, there were some initial struggles to define the peer mentor role and relationship to others on the IPS team.

\section{Perceptions of Peer Mentoring}

\section{What did mentees think about peer mentoring as part of IPS?}

A survey found that most mentees benefitted from peer mentoring. Mentees valued experiences of trust, feeling understood, and forming a meaningful relationship through talking one-on-one. Valued mentor characteristics included authenticity, flexibility, and being a YAP graduate.

\section{The Value of Vocational Peer Mentors}

"[My peer mentor and I] talked about my job and how important it was that I keep it...but it was also a bond."

"[My peer mentor] understands where I am coming from and I understand where she is coming from."

"[My peer mentor] didn't look at me differently...she didn't put on a phony act."

${ }^{1}$ Bond, G. R. (2004). Supported employment: Evidence for an evidence-based practice. Psychiatric Rehabilitation Journal, 27(4): $345-359$.

University of Massachusetts UMASS. Medical Schoo

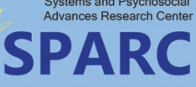

\section{THRESHOLDS}

The contents of this tip sheet were developed with funding from the US Department of Education, National Institute on Disability and Rehabilitation Research, and the Center for Mental Health Services, Substance Abuse and Mental Health Services Administration (NIDRR grant H133B090018). Additional funding provided by UMass Medical School's Commonwealth Medicine division. The content of this tip sheet does not necessarily reflect the views of the funding agencies and you should not assume endorsement by the Federal Government.

The Transitions RTC is part of the Systems \& Psychosocial Advances Research Center (SPARC) A Massachusetts Department of Mental Health Research Center of Excellence
Suggested Citation: Vorhies,V., Sabella, K., Fagan, M., Ellison, M. \& Davis, M. (2014). Using Peer Mentors in Career Programs for Transition Aged Youth with Mental health Conditions. Worcester, MA: University of Massachusetts Medical School, Department of Psychiatry, Systems and Psychosocial Advances Research Center (SPARC), Transitions Research and Training Center.

This publication can be made available in alternative formats upon request through TransitionsRTC@umassmed.edu

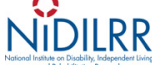

